\title{
PRESENTACIÓN: EXPLORANDO INTERSECCIONES ENTRE RELIGIÓN Y PRÁCTICAS Y TEORÍAS EDUCATIVAS EN ESCENARIOS CANADIENSES Y EUROPEOS
}

\section{Presentation: exploring intersections between religion and practices and educational theories in Canadian and European scenarios}

\author{
Rosa BRUNO-JOFRÉ \\ Queen's University \\ Correo-e: brunojor@queensu.ca \\ Patricia Quiroga Uceda \\ Investigadora independiente \\ Correo-e: patquceda@gmail.com
}

Os EsTUdios MONOGRÁficos que presentamos están dedicados a seis es-
cenarios que ilustran intersecciones entre educación y religión en tiempos
históricos y espacios diferentes. Los dos primeros artículos se centran en
el papel de dos congregaciones enseñantes femeninas y sus respuestas al contexto
misionero: las Hermanas de la Caridad (Sisters of Charity of St. Vincent de Paul),
dedicadas a la salud, la educación, el ministerio pastoral y los servicios sociales
con la casa madre, en Halifax, Nueva Escocia, desde i849, y la provincia española
de las Religiosas del Niño Jesús, St. Maur, congregación de origen francés funda-
da en i662 y dedicada a la enseñanza.
Este número abre con «Arts, crafts, and rural rehabilitation: The Sisters of
Charity, Halifax, and vocational education in Terence Bay, Nova Scotia, I938-
I942», escrito por las historiadoras Sasha Mullally y Heidi MacDonald. El artí-
culo se sitúa temporalmente entre los años ig38 y i943 en la provincia de Nueva
Escocia, Canadá, en la región del país conocida como las Marítimas. La congre-
gación respondió a la pobreza rural que las rodeaba como consecuencia no sólo 
PRESENTACIÓN: EXPLORANDO INTERSECCIONES ENTRE RELIGIÓN Y PRÁCTICAS Y TEORÍAS EDUCATIVAS EN ESCENARIOS CANADIENSES Y EUROPEOS ROSA BRUNO-JOFRÉ Y PATRICIA QUIROGA UCEDA

de la Gran Depresión, sino del declive de la pesca y el proceso de industrialización y crearon un centro de artesanías. Las hermanas lo describían como un centro de rehabilitación social y económica. Se trataba de programas de entrenamiento, de carácter comunitario, en tejido y carpintería, sirviendo de modelo para la supervivencia de los pueblos costeros.

La intersección de las hermanas con las condiciones locales de pobreza y las configuraciones dominantes eran mediadas no sólo por su llamado religioso, pero necesariamente convergía con agendas políticas que tenían raíces e intencionalidades que pueden ser construidas como divergentes. El proyecto estaba financiado por el adinerado senador canadiense William Dennis y apoyado por el Rev. Moses Coady, fundador del Movimiento Antigonish y conocido por promover el movimiento cooperativista y el desarrollo comunitario local. El proyecto, que, según las autoras, fue diseñado de un modo que pudiera generar una sensibilidad hacia un nuevo orden liberal, no consiguió responder a las necesidades de la comunidad y no pudo corregir las raíces de la pobreza.

El segundo artículo se titula «Las religiosas del Niño Jesús en Bembibre, León, España, en la segunda etapa del franquismo: la escuela sin puertas», escrito por Rosa Bruno-Jofré, historiadora e historiadora de la educación. El artículo examina la transformación que vive la congregación, originariamente francesa, como consecuencia de la coyuntura histórica en la que convergen a nivel transnacional, los cambios culturales de los largos años sesenta, un profundo proceso de secularización, una visión pluralista del mundo y el Vaticano II. A nivel local, España se encuentra bajo el segundo estadio del franquismo, con un movimiento hacia un enfoque tecnocrático, mientras que sectores de la Iglesia reaccionan en contra del régimen. Vaticano II lo impacta. Un claro ejemplo es la cuestión de la libertad religiosa en un Estado confesional. Tratándose de una congregación enseñante, los cambios generados por la Ley de Educación de 1970 y la política tecnocrática desarrollista favorecieron la financiación de las escuelas. Sin embargo, los cambios que se dieron en el proceso de renovación religiosa empujado por Perfectae Caritatis (documento del Vaticano II), de resignificación de la visión y misión de la congregación dieron lugar a cambios radicales que generaron una relación abierta, pluralista, incluyente entre la escuela y la comunidad. La radicalidad del cambio tuvo mucho que ver con el proceso motivado por Perfectate Caritatis, que exigió a las congregaciones volver a sus raíces, a la inspiración inicial de los fundadores. El fraile Mínimo Nicolás Barré fundó Las Religiosas del Niño Jesús (originariamente Instituto de Maestras Caritativas) en un ejercicio de claro desafío a los límites impuestos por el papado a las mujeres consagradas, en medio de un ambiente de controversia y renovación espiritual en el siglo xvir. Metodológicamente la autora usa un enfoque transtemporal para descubrir conexiones, buscar comparaciones y examinar la fuente de la dirección tomada por las Religiosas del Niño Jesús en España en los años 1960 y 1970'.

Armitage, David: «What's the Big Idea? Intellectual History and the Longue Durée», History of European Ideas, 38, 4 (2012), pp. 493-507. 
PRESENTACIÓN: EXPLORANDO INTERSECCIONES ENTRE RELIGIÓN Y PRÁCTICAS Y TEORÍAS EDUCATIVAS EN ESCENARIOS CANADIENSES Y EUROPEOS ROSA BRUNO-JOFRÉ Y PATRICIA QUIROGA UCEDA

Es evidente que los apostolados activos, históricamente específicos de las congregaciones misioneras se ubican en un contexto de fuerzas macropolíticas que interactúan a nivel internacional, están insertas en las políticas de la Santa Sede y se cruzan con los intereses y deseos locales, agendas políticas y económicas, resistencias y maneras de vivir la fe. Los giros teológicos que convergieron en el Vaticano II abrieron puertas para un encuentro con el mundo en términos nuevos y desde un posicionamiento relativo de apertura al mundo, que invitó a la «contaminación cognitiva» (el pluralismo produce la contaminación cognitiva y esta última la relativiza), conceptos que desarrolla Peter Berger para explicar su paradigma de pluralismo y que Bruno-Jofré adopta para explicar cambios en la vida y visión de las congregaciones ${ }^{2}$. No toda la fuerza explicativa se centra en los procesos de secularización per se.

El enfoque que desarrollaron las Religiosas del Niño Jesús en su apostolado en Bembibre, León, convirtiendo la escuela en un centro comunitario, afirmando su autonomía frente al clero local pero trabajando juntos, abriéndose a la comunidad de manera incluyente, así como la noción de escuela popular, abierta a la calle, sin puertas tuvo su punto de inspiración en el ideario de su fundador $\mathrm{Ni}$ cholas Barré y sus raíces en el espíritu de renovación del siglo xvir que convergió de alguna manera con los cambios motivados por el concilio Vaticano II.

Uno de los temas más controvertidos en la historia de la educación católica es la cuestión pedagógica, en particular la recepción de parte de los educadores católicos de métodos activos, de los principios de la escuela nueva y, sobre todo, del pensamiento de John Dewey. Desde una perspectiva filosófica, el pensamiento pragmatista deweyano ${ }^{3}$, en particular el falibilismo (incertidumbre inevitable) entraba en conflicto con la noción de la Iglesia como depositaria de la verdad aun cuando la libertad de conciencia era valorada. El rechazo de Dewey al dualismo (central en la teología católica a menudo expresado en la dualidad cuerpo y alma) no era menos problemático. La solución era bastante extendida. Se dejaba de lado la base filosófica pragmatista y se apoyaban los proyectos pedagógicos en un Dewey «mutilado» y en los trabajos de W. Kilpatrick, aunque Dewey era a menudo un punto legitimador de referencia ${ }^{4}$. Las escuelas católicas que lo aplicaban estaban advertidas de las implicaciones de la dimensión participativa, pero lo veían en relación a un fin educacional holístico y al desarrollo subjetivo. Uno de los casos más conocidos es el de la Corpus Christi School en la ciudad de Nueva York, ubicada frente al Teachers College de la Columbia University, a cargo de las Dominican Sisters de Sinsinawa, donde la figura del padre George M. Ford,

\footnotetext{
Berger, Peter: The Many Altars of Modernity: Toward a Paradigm for Religion in a Pluralist Age, Boston \& Berlin, DeGruyter, 20I4, chapter I.

3 Bruno-Jofré, R. y Jover, G.: «The Readings of John Dewey's Work and the Intersection of Catholicism: The Cases of the Institución Libre de Enseñanza and the Thesis of Father Alberto Hurtado, S.J. on Dewey», en Bruno-Jofré, R. y Schriewer, J. (eds.): The Global Reception of John Dewey's Thought: Multiple Refractions Through Time and Space, New York \& London, Routledge, 20I2, pp. 23-42.

4 La expresión Dewey «mutilado» es usada en Bruno-Jofré y Jover (2OI2).
} 
PRESENTACIÓN: EXPLORANDO INTERSECCIONES ENTRE RELIGIÓN Y PRÁCTICAS Y TEORÍAS EDUCATIVAS EN ESCENARIOS CANADIENSES Y EUROPEOS ROSA BRUNO-JOFRÉ Y PATRICIA QUIROGA UCEDA

su pastor, quien había sido estudiante en el Teachers College, alcanzaba un perfil nacionals. La escuela tenía una dimensión democrática que buscaba desarrollar a través de una pedagogía activa, avalada por una lectura mediada por el contexto local de la Encíclica sobre la Educación Cristiana (1929) del papa Pío XI. También el jesuita chileno Alberto Hurtado había reconocido la importancia de esa dimensión educativa. Sin embargo, las intencionalidades en juego en el proceso de aplicación de una pedagogía activa en el contexto de la España franquista, que es el tema del artículo siguiente, es mucho más complejo.

Carlos Martínez Valle es el autor de «Secularización social y métodos activos para la catequesis escolar en el franquismo (1939-1975): problemas y efectos de una transferencia de praxis educativa», un artículo que examina, dada su ubicación en el régimen franquista, un elemento más: el nacionalcatolicismo. Martínez Valle, formado en ciencias políticas, examina con riqueza interpretativa el interés en la renovación de los métodos pedagógicos en relación a la enseñanza de la religión, que en realidad se trataba de catequesis. El currículum respondía a las características del Estado confesional y la enseñanza de la religión, confesional, doctrinaria, era una materia escolar. Lo interesante en este artículo es que el redescubrimiento de la Escuela Nueva durante el franquismo y los debates sobre los métodos activos aplicados en la catequesis escolar se iniciaron en los años cuarenta en círculos académicos familiarizados con John Dewey, así como con representantes de la Escuela Nueva. Sin embargo, los catequistas de base, que sintieron el impacto de la secularización y del Vaticano II y vieron la necesidad de modificar la metodología pedagógica para enseñar la doctrina de la Iglesia como parte de un magisterio pastoral, adoptaron métodos activos procedentes de la tradición social de la Iglesia tales como la Revisión de vida y los círculos de debate, métodos con una fuerte dimensión democrática. No acudieron a los métodos activos de la tradición escolanovista sino a los métodos propios de la tradición social de la Iglesia. Martínez Valle resalta en su argumento la capacidad de las prácticas de modelar y transformar ideas en el contexto de una concienciación y práctica social que, inspiradas por el Vaticano II, reivindicaba una pastoral social y se ligaba a la vida de los necesitados.

Los «largos años sesenta» ${ }^{6}$ en Ontario fueron años de un encuentro intenso con la modernidad en el contexto de un proceso de secularización y la presencia de un pluralismo que sienta un tono diferente para la educación. Canadá recreaba su identidad como un país moderno, global, aunque reteniendo lazos residuales con su pasado colonial británico. Los cambios políticos, económicos y tecnológicos que tuvieron lugar en Estados Unidos, Gran Bretaña y Europa, así como el realineamiento que se produjo con la Guerra Fría, tuvieron implicaciones para la educación. Ciertamente, la Iglesia católica participó en la batalla ideológica entre el capitalismo y el comunismo soviético. Es importante mencionar en relación

Ford, George Barry: A Degree of Difference, New York, Farrar, Straus \& Giroux, 1969.

La expresión «los largos años sesenta» que va de 1958 a 1974 es tomada de Marwick, Arthur: The Sixties: Cultural revolution in Britain, France, Italy and the United States, c. 1958-1974, Oxford, Oxford University Press, 1998. 
PRESENTACIÓN: EXPLORANDO INTERSECCIONES ENTRE RELIGIÓN Y PRÁCTICAS Y TEORÍAS EDUCATIVAS EN ESCENARIOS CANADIENSES Y EUROPEOS ROSA BRUNO-JOFRÉ Y PATRICIA QUIROGA UCEDA

al artículo que sigue en esta colección que las transformaciones tecnológicas continuaron después de la Segunda Guerra Mundial y establecieron el escenario para la globalización económica. De especial relevancia para la educación fue el desarrollo de tecnologías de comunicación tales como la televisión que revolucionaron la construcción de las subjetividades populares 7 . Josh Cole en su artículo " $" O u r$ only hope is Apocalypse": Marshall McLuhan, Catholic antimodernism, and I96os education reform», nos lleva a la provincia de Ontario, Canadá, a una década de cambios profundos en educación y en la sociedad, una fuerte apertura hacia la modernidad y la conjunción con el Vaticano II, que habría de generar cambios en la vida de los católicos y en el sistema católico. El artículo se centra en el teórico canadiense, católico, Marshall McLuhan, quien en los sesenta había alcanzado reputación internacional con sus observaciones sobre la educación y los medios de comunicación. En un tono semejante a Ivan Illich hablaba de la disolución de las escuelas y cuestionaba la relevancia de los trabajadores de la educación. Su crítica a la modernidad era también construida como vanguardia, aunque, del mismo modo que Illich, su pensamiento crítico tenía raíces antimodernistas y se inspiraba en un premodernismo cristiano bastante vago. Parece paradójico que, en los años sesenta, el Gobierno conservador de Ontario, que inauguró un periodo de modernización en reforma educativa sin paralelismo en el resto del país, consultara intensamente a McLuhan. A partir de los años noventa, después de décadas de oscuridad renace su presencia en un mundo postmodernista, tal como sucede con Illich .

El artículo de Joe Stafford, titulado «Looking to the past and moving to the future: a catholic high school religious curriculum for the $2 \mathrm{I}^{\mathrm{st}}$ century», se ubica también en la provincia canadiense de Ontario y se centra en el currículum de educación religiosa a nivel secundario en las escuelas del sistema católico en esa provincia. El autor trata de explicar las razones que se encuentran detrás de la pobre preparación religiosa de los alumnos y alumnas del sistema católico a través del análisis del contenido, así como de la metodología utilizada en la enseñanza religiosa. Para ello, Stafford contextualiza los problemas históricamente partiendo en los largos años sesenta y en la llamada 'crisis religiosa' dentro de un proceso acelerado de secularización, el distanciamiento entre los liberales y los conservadores y la laicización de las escuelas católicas. Las teorías de la secularización son de utilidad en este caso porque, si bien han sido criticadas por su uso extensivo para explicar cambios, se aplican bien al colapso de la cultura y práctica

Bruno-Jofré, R. y Cole, J.: «What the Dead Say to the Living: Time, Politics, and Teacher Preparation in English Canada's Long-I96os", en Horlacher, R. y Hoffmann-Ocon, A. (eds.): Pädagogisches Wissen in der Lebrerinnen-und Lebrerbildung [Educational Knowledge in Teacher Education], Germany, Klinhardt, 2016.

8 Bruno-Jofré, Rosa e Igelmo Zaldívar, Jon: «The Center for Intercultural Formation, Cuernavaca, Mexico its Reports (1962-1967) and Ivan Illich's Critical Understanding of Mission in Latin America», Hispania Sacra, LXvi, extra II (20I4), pp. 457-487. Bruno-Jofré, Rosa e Igelmo ZaLdívar, Jon: «Ivan Illich's Late Critique of Deschooling Society: "I was Largely Barking up the Wrong Tree" ", Educational Theory, Journal of the John Dewey Society and the Philosophy of Education Society, 62, 5 (2012), pp. 573-592. 
PRESENTACIÓN: EXPLORANDO INTERSECCIONES ENTRE RELIGIÓN Y PRÁCTICAS Y TEORÍAS EDUCATIVAS EN ESCENARIOS CANADIENSES Y EUROPEOS ROSA BRUNO-JOFRÉ Y PATRICIA QUIROGA UCEDA

cristiana particularmente en Europa, Norteamérica y Australasia. Stafford introduce un elemento de análisis nuevo al discutir el proceso de «secularización de la conciencia» que tuvo lugar en las escuelas católicas al adoptar valores seculares sin ni siquiera darse cuenta.

Stafford cuestiona el documento curricular para la educación religiosa de 2006, al cual caracteriza fundamentalmente como un documento de catequesis, asumiendo que los estudiantes poseen el conocimiento y entendimiento necesarios. Así, se deja de lado la diferenciación entre catecismo y educación religiosa a pesar de las diferentes demandas generadas por dichos enfoques. La propuesta que hace, inspirada por el trabajo de William Pinar, en particular, su teoría de la subjetividad y el desarrollo de sí mismo (self), es la de un currículo basado en educación religiosa (no catequética). Propone un currículo de educación religiosa que reconozca la influencia de la cultura secular y que sea académicamente riguroso, abra paso a preguntas, análisis, dudas y en el que los Evangelios sean examinados como documentos históricos.

Esta colección monográfica concluye con un artículo de Daniel Tröhler, «Los fundamentos religiosos de la Bildung como epítome de la educación alemana y su fundamental recelo antioccidental», que examina la construcción de la idea educativa de Bildung: una autorrealización interna del alma que no acude a las disciplinas modernas tales como la ciencia, la política o la economía y que, Tröhler argumenta, tiene sus raíces en las maneras de pensar luteranas. El dualismo, que Tröhler ubica en el lenguaje educativo luterano (por ejemplo, empirismo y espíritu; pluralidad [realidad externa] y unidad; interior y exterior), lleva a una diferenciación entre educación vocacional y educación general ${ }^{9}$. El artículo recorre históricamente el desarrollo filosófico que contextualiza a Bildung y la dimensión política de la ideología de Bildung y sus implicaciones clasistas para la educación, así como los nuevos desarrollos en educación científica que se generan desde finales del siglo XIX. Durante la Gran Guerra, bajo la República de Weimar, la integración de la educación como disciplina en la universidad, como carrera, se da bajo el signo de Bildung, con la mirada en el mundo ideal de la antigüedad, pero solidificando la idea de que la cultura alemana era la verdadera heredera de los griegos. Más allá de los movimientos de reforma de la educación y sus implicaciones clasistas, existía la idea de una unidad dada por el nuevo ideal de la persona alemana. El relato histórico de Tröhler nos lleva a desarrollos contemporáneos. Críticos de los informes PISA, los que según Tröhler caerían dentro de una concepción calvinista, no luterana como Bildung, usan conceptos derivados de la tradición Bildung en sus argumentos. Podemos decir a modo de conclusión, parafraseando a Tröhler, que la reintroducción de la teoría de Bildung sin un proceso de reinterpretación de las categorías binarias de alta y baja socialización y la creación de una relación de unidad categórica no tiene implicaciones conceptuales.

9 Horlacher, Rebekka: «Qué es Bildung? El Eterno Atractivo de un Concepto Difuso», Pensamiento Educativo, Revista de Investigación Educational Latinoamericana, 5I, I (2014), pp. 3545. TröHler, Daniel: Languages of Education: Protestant Legacies, National Identities, and Global Aspirations, New York \& London, Routledge, 2011, pp. I48-163. 
PRESENTACIÓN: EXPLORANDO INTERSECCIONES ENTRE RELIGIÓN Y PRÁCTICAS Y TEORÍAS EDUCATIVAS EN ESCENARIOS CANADIENSES Y EUROPEOS ROSA BRUNO-JOFRÉ Y PATRICIA QUIROGA UCEDA

Este monográfico ha reunido a historiadores/as, quienes presentan sus investigaciones sobre temas y casos específicos en los que la intersección de la religión con la educación no puede separarse de posicionamientos filosóficos, políticos, y económicos que le sirven como referentes. Sin embargo, tampoco pueden reducirse a ellos. Las presentes investigaciones se basan en estudios de fuentes y en ocasiones forman parte de trabajos de investigación a largo plazo. Esperamos que los lectores encuentren elementos nuevos que enriquezcan el debate temático y también historiográfico en historia de la educación. 